\title{
Green Refining of Waste Lubricating Oil: A China Perspective
}

\author{
Jinlong $\mathrm{Wu}^{1}$, Bo Li ${ }^{1}$, Wei Wang ${ }^{1}$, Shu Yang ${ }^{1}$, Peng Liu ${ }^{1}$, Bo Zhang ${ }^{1, *}$, Changyan \\ Yang $^{1,2, *}$, Yigang Ding ${ }^{1}$ \\ 1: Key Laboratory for Green Chemical Process of Ministry of Education, Hubei Key Laboratory of Novel \\ Chemical Reactor and Green Chemical Technology, School of Chemical Engineering and Pharmacy, \\ Wuhan Institute of Technology, Hubei, China \\ 2: Hubei Key Laboratory for Processing and Application of Catalytic Materials, Huanggang Normal \\ University, Hubei, China
}

Received January 5, 2019; Accepted April 3, 2019; Published April 25, 2019

Presently, many regeneration processes of waste lubricating oil, such as catalytic hydrogenation, are available. However, some of these processes are highly costly and not suitable for Chinese economic conditions, and some may produce contaminated impurities such as acid slag, which cannot meet environmental protection requirements. This study aims to develop a green process for the regeneration of waste lubricating oil into a base oil, which should meet the requirements of green chemistry, have the characteristics of simple operation, low cost, less pollution and high recovery rate, and turn wastes into renewable resources. The new process developed via this research has three stages. First, mechanical and large particle impurities in the waste lubricating oil were removed by pretreatment. Second, most of the colloid and asphaltene were removed by thermal extraction and sedimentation. Finally, the activated bleaching earth was used to further purify the waste lubricating oil. The performance evaluation of the finally obtained lubricating base oil conformed to the standard of the HVI-100 lubricating oil. The total recovery rate of the process was about $63.5 \%$.

Keywords: Waste lubricating oil; Green refining; Pretreatment; Thermal extraction and sedimentation; Response surface methodology

\section{Introduction}

\subsection{Overview}

The development of the lubricant industry is directly linked to the development of the transportation industry and has a greater impact on the ups and downs of the macro economy. From 2000 to 2010, along with the rapid development of China's economy, the scale of the lubricant market has continued to grow rapidly. In 2010, the consumption of lubricating oil reached 8.5-8.8 million tons/year [1]. Since 2013, China's economic development has entered a steady growth phase. Many industries including the lubricant industry have been affected and showed a gradual slowdown in development. The lubricant industry in China has been continuously eliminating outdated products and switching to high-end products with the extended oil-changing period.

In 2017, the domestic demand for automotive lubricants was 3.572 million tons, accounting for $53 \%$ of the total lubricant market, and the share of industrial ones was reduced to $28 \%$ [2]. Lubricant or motor oil is a consumer product for all vehicles, which 
needs to be replaced every $5000-7000 \mathrm{~km}$ or $3000-5000$ miles. Correspondingly, the amount of waste lubricating oil produced in China is proportional to this market demand (Figure 1) [3].

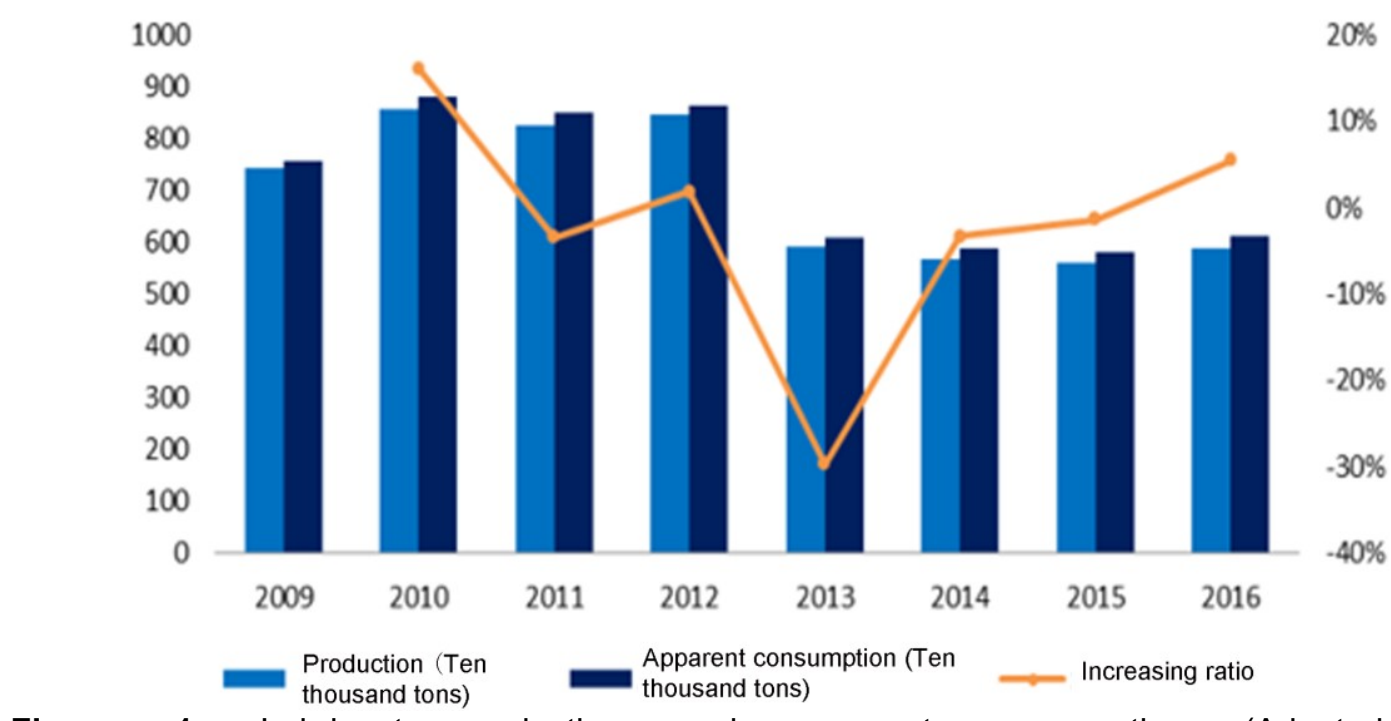

Figure 1. Lubricant production and apparent consumption (Adapted from http://www.chyxx.com/industry/201708/555496.html, Public Domain)

During the use, some hydrocarbons in the lubricating oil will react with oxygen at high temperature and pressure to produce peroxides, carboxylic acids, alcohols, ketones, asphaltenes, gums, etc. The color of the oil becomes darker [4]. The produced acids cause the increased acidity of the lubricating oil, which can corrode easily the mechanical parts, resulting in the reduced service life. Meanwhile, incomplete combustion in the engine produces carbon residue, and mutual friction between mechanical parts tends to generate minute metal powder particles. Dust, moisture, and soil in the environment are also components of impurities in the lubricating oil. These impurities may form deposits on the surface of mechanical parts, which are liable to cause engine blockage and reduce its service life [5]. When the metamorphic portion reaches $5-10 \%$, the lubricating oil must be disposed of as waste lubricating oil.

Waste lubricating oil is mainly composed of base oil, asphaltene, colloids, oxides, carbon residue, sludge, moisture, and other components [6]. The main oxides in waste lubricating oils are carboxylic acids, carboxylates, and aldehydes [7]. Metals such as Ni, $\mathrm{Sn}, \mathrm{Cr}$, and $\mathrm{Fe}$ are produced by corrosion of the engine, while metals like $\mathrm{Ag}, \mathrm{B}, \mathrm{Ba}, \mathrm{Ca}$, $\mathrm{Cd}, \mathrm{Co}, \mathrm{Hg}, \mathrm{Mg}, \mathrm{Mo}, \mathrm{P}, \mathrm{Pb}, \mathrm{Sb}, \mathrm{Se}, \mathrm{Ti}, \mathrm{Zn}$, etc. are derived from the additives [8]. Waste lubricating oil is mainly processed in the following ways [9-12]:

(1) Directly discarded as wastes;

(2) Catalytic cracking;

(3) Used as a fuel after removing heavy metals;

(4) Raw materials for road oiling;

(5) Regeneration into a lubricating base oil

Among them, the regeneration of waste lubricating oil into a base oil has better economics and reflects the concept of green chemistry. As early as the beginning of the 20 th century, the recycling process of waste lubricating oil was commercialized on a large scale in the United States and Germany. China has carried out research on the 
regeneration process of waste lubricating oil since the 1940s. So far, most of China's manufacturers are still using the sulfuric acid-bleaching earth process, which was mainly used in the early 1960s by other countries. Currently, petroleum has become an increasingly important resource, and environmental protection became an unavoidable issue. Many countries have raised the standard for the recycling of waste lubricating oil and issued the policies to encourage the recycling.

\subsection{Regeneration Processes of Waste Lubricating Oil}

\subsubsection{Sulfuric Acid - Bleaching Earth Process}

The sulfuric acid-bleaching earth process (Figure 2) utilizes the principle that properties of oil and water are incompatible, and the boiling point of water is lower than that of waste oil. [13, 14]. The water is first removed by heating. Then, concentrated sulfuric acid is used to oxidize organic matters, which is removed by washing with alkali. The active bleaching earth absorbs impurities such as pigments. Final filtration could remove solid residues.

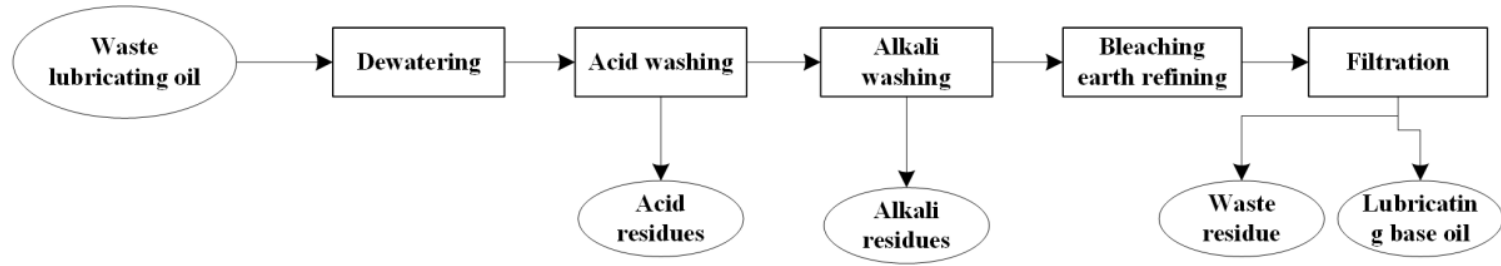

Figure 2. Sulfuric acid- bleaching earth process

\subsubsection{Hydrotreating}

The hydrotreating process has advantages including the high oil yield, simple operation and well-established process, but its cost is relatively high [15]. It is widely used abroad. Under certain conditions of catalysis and pressure, impurity elements such as $\mathrm{N}, \mathrm{O}$, S, etc. in the waste oil are removed as their hydrides such as $\mathrm{NH}_{3}, \mathrm{H}_{2} \mathrm{O}, \mathrm{H}_{2} \mathrm{~S}$, etc [16]. The olefin and aromatic hydrocarbons can be partially hydrogenated to improve the quality of the oil.

\subsubsection{Molecular Distillation}

The mass transfer and heat transfer of the device for molecular distillation is very efficient, and the material requirements are relatively demanding. It is necessary to remove the pollutants such as mechanical impurities in the waste lubricating oil before further purification (Figure 3). The quality of the lubricating oil obtained by the molecular distillation process $[4,17]$ satisfies the national standard of the lubricating base oil. But the distillation process has higher requirements on the environment and requires a higher degree of vacuum. The size of the reactor is fixed and has certain limitations. 


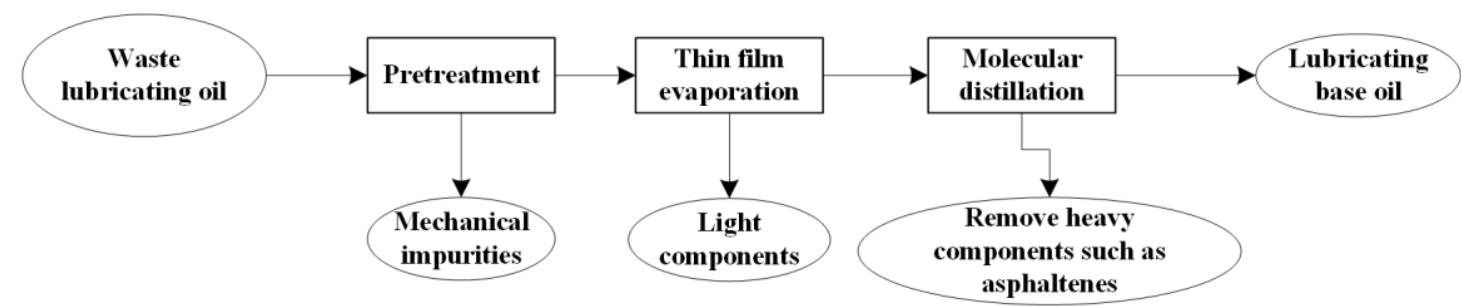

Figure 3. Molecular distillation process

\subsubsection{Solvent Refining}

Solvent refining is one of the commonly used methods for refining petroleum products. Figure 4 shows the solvent refining process flow chart. It utilizes different solubility of components of the lubricating oil in a solvent to remove impurities and nonideal component $[18,19]$.

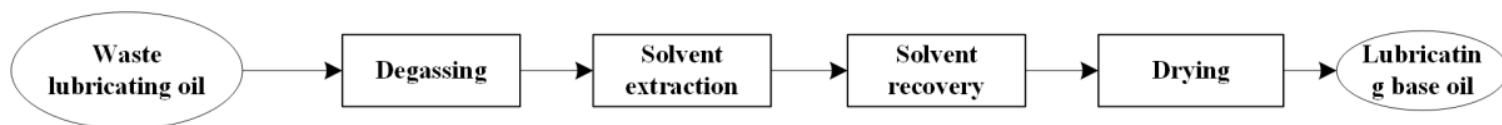

Figure 4. Solvent refining process

The degassing process removes oxygen dissolved in the oil to prevent the solvent from being slowly oxidized. After solvent extraction, two layers are formed. The lower layer is a mixture of solvent and impurities, and the upper layer mainly contains refined oil. The energy consumption of the solvent recovery part accounts for $80 \%$ of the process. The solvent in the upper layer is removed in the evaporation stripper, and then dewatered to obtain the lubricating base oil.

\subsubsection{Membrane Separation}

Recently, membrane separation process [20] has been proposed as new mean to regenerate waste lubricating oil. Because of its high cost, related studies remain at the research stage. Figure 5 is a flow chart of the membrane separation process.

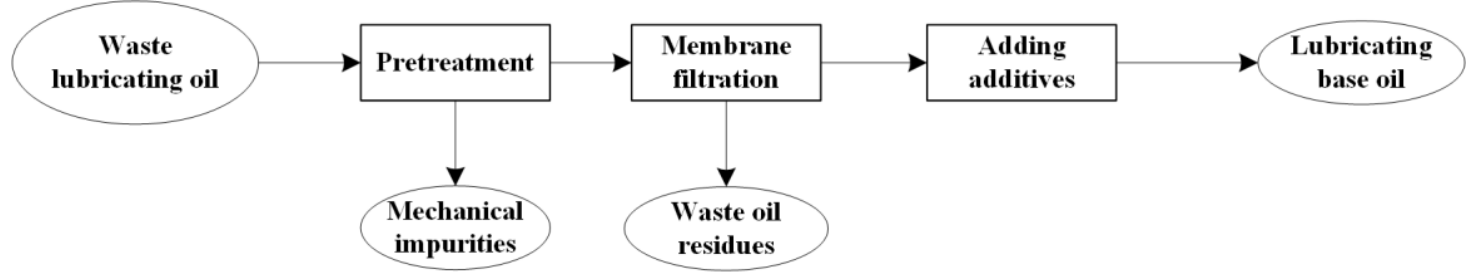

Figure 5. Membrane separation process

\subsection{The Choice of Waste Lubricating Oil Regeneration Process in China}

The hydrofining process is used by many developed countries for waste lubricant treatment. However, the investment in hydrotreating process is relatively high. For the time being, there is no waste lubricating oil recycling plant in China that uses hydrotreating process.

The problems with the solvent refining process are the loss of the solvent, the low base oil yield, and the high sulfur content of the base oil product. At present, the solvent formulation has been improved, and the consumption of the solvent has been significantly reduced. Compared with the hydrofining process, the investment cost of the solvent refining plant is lower, and the technology is more mature. 
The sulfuric acid-bleaching earth process is mature and requires relatively low investment cost. Many domestic refineries are using this method. However, the residual acid slag and alkali slag have a great impact on the environment. With the gradual strict environmental policy in China, the process will gradually be eliminated by the market.

\section{Materials and Methods}

\subsection{Materials}

The waste lubricating oil used in this study was provided by Tianan Chemical Co., Ltd (Macheng, Hubei Province, China). The color of the waste lubricating oil is carbon black, which is very thick at room temperature, and has an unpleasant pungent odor. Its properties are listed in Table 1.

Table 1. Performance evaluation results of the waste lubricating oil

\begin{tabular}{ccc}
\hline Property & Value & Method \\
\hline Viscosity $(\mathrm{mPa} \cdot \mathrm{s}) / 40^{\circ} \mathrm{C}$ & 78.50 & LVDV-1 viscometer \\
Acid value $(\mathrm{mg} \cdot \mathrm{KOH} / \mathrm{g})$ & 0.59 & $\mathrm{~GB} / \mathrm{T} 264-1983$ \\
Moisture $(\%)$ & 0.55 & $\mathrm{~GB} / \mathrm{T} 260-2016$ \\
Mechanical impurity $(\%)$ & 1.42 & $\mathrm{~GB} / \mathrm{T} 511-2010$ \\
Ash $(\%)$ & 0.57 & $\mathrm{~GB} / \mathrm{T} 508-1985$ \\
\hline
\end{tabular}

The chemical reagents of isopropanol, n-butanol, isobutanol, and butanone were purchased from Sinopharm Chemical Reagent Co., Ltd (Shanghai, China).

Table 2. Properties and prices of alcohol and ketone solvents*

\begin{tabular}{ccccc}
\hline Solvent & Isopropanol & n-butanol & Isobutanol & Butanone \\
\hline Boiling point $\left({ }^{\circ} \mathrm{C}\right)$ & 82.5 & 117.7 & 108.1 & 75.9 \\
Freezing point $\left({ }^{\circ} \mathrm{C}\right)$ & -88.5 & -90.2 & -108.0 & -85.9 \\
Price $(¥ \mathrm{YMB} /$ ton$)$ & 7800 & 7600 & 7400 & 7500 \\
\hline
\end{tabular}

${ }^{*}$ All solvents listed in Table 2 have intermediate toxicity and stable at room temperature.

The activated bleaching earth (also called activated clay) was provided by Zhengzhou Fengquan Water Treatment Plant (Henan, China). The physical and chemical properties of the products are shown in Table 3.

Table 3. Physical properties and composition of the activated bleaching earth

\begin{tabular}{cc|cc}
\hline Property & Index & Composition & Weight $\%$ \\
\hline Appearance & White fine powder & $\mathrm{SiO}_{2}$ & 62.34 \\
Surface area & $>130 \mathrm{~m}^{2} / \mathrm{g}$ & $\mathrm{Al}_{2} \mathrm{O}_{3}$ & 17.24 \\
Moisture & $<5 \%$ & $\mathrm{Fe}_{2} \mathrm{O}_{3}$ & 2.73 \\
Decolorization & $>154$ & $\mathrm{FeO}$ & 0.12 \\
Heavy metal content & $<10 \mathrm{mg} / \mathrm{kg}$ & $\mathrm{TiO}$ & 0.15 \\
Arsenic content & $<3 \mathrm{mg} / \mathrm{kg}$ & $\mathrm{CaO}$ & 2.09 \\
Free acid & $<0.1 \%$ & $\mathrm{MgO}$ & 5.44 \\
Granularity $(<0.076 \mathrm{~mm})$ & $>95 \%$ & $\mathrm{MnO}$ & 0.15 \\
\hline
\end{tabular}


The activated bleaching earth was dried in an oven at $110^{\circ} \mathrm{C}$ for 2 hours prior to the experiments.

\subsection{Characterization of Lubricating Oil}

Viscosity was measured by using the LVDV-1 rotary electronic viscometer (Shanghai Jingtian Electronic Instrument Co., Ltd, China). The acid value was measured by the indicator titration method according to the national standard GB/T264-1983. The moisture content of the oil was measured by the distillation method according to the national standard GB/T260-2016, while the ash content was determined according to the national standard GB/T508-85.

Mechanical impurities in the oil refer to solid small particles that cannot be dissolved in a solvent such as toluene or gasoline. To measure mechanical impurities, the oil was first dissolved in the toluene, which was followed by filtration (GB/T511-2010).

Color of the oil (i.e, chromaticity) could reflect the degree of refinement and its stability. A lighter oil color generally indicates a higher degree of purification of the waste lubricating oil [21]. The color of the recycled oil was determined according to ASTM D1500-12. The sample oil and standard swatches are placed under a certain light source, and the color is compared to determine the ASTM color of the oil.

\subsection{Thermal Settlement}

Thermal settling is used as a pretreatment method, which is an effective physical method of removing the mechanical impurity from a large volume of waste lubricating oil, which has the advantages of low energy consumption and easy handling. For each test, about $60 \mathrm{ml}$ of waste lubricating oil was added to a $100 \mathrm{ml}$ beaker, which was placed into an oven at $40-80{ }^{\circ} \mathrm{C}$ for 3-24 h. The mechanical impurity content was measured after each test.

\subsection{Thermal Extraction and Sedimentation}

Solvents such as short-chain alcohols and ketones have poor dissolving ability for substances with relatively high molecular weight such as asphaltenes, colloids, gray matter and additives, but strong dissolving ability for oil components. Heating can reduce the viscosity of the waste lubricating oil, making the extraction process more complete. Therefore, high molecular weight substances settle down during thermal extraction and sedimentation. Commonly used extractants are polar solvents such as C3 or C4 alcohols and butanone, which showed good solubility to the waste lubricating base oil $[22,23]$.

Considering the cost, solvents of isopropanol, n-butanol, isobutanol, and butanone were used for comparison.

\subsection{Response Surface Methodology for Thermal Extraction and Sedimentation using Double Solvents}

To further analyze the effect of using two extraction solvents on thermal extraction flocculation, the response surface methodology was used to design the experiments (Table 4). 
Table 4. Box-Behnken response surface test design factors and levels

\begin{tabular}{cccc}
\hline Level & A: solvent to oil & B: solvent ratio & C: Temperature $\left({ }^{\circ} \mathrm{C}\right)$ \\
\hline-1 & $2: 1$ & $1: 4$ & 30 \\
0 & $4: 1$ & $2.13: 1$ & 50 \\
1 & $6: 1$ & $4: 1$ & 70 \\
\hline
\end{tabular}

The yield of waste lubricating oil is the response value, and three factors of A, B, and $\mathrm{C}$ are the ratio of solvent to oil (A: the volume ratio of the extractant: mass of crude lubricating oil), the solvent ratio (B: the volume ratio of n-butanol: isobutanol), and temperature $(\mathrm{C})$, respectively. This three-factor three-level response surface analysis included 17 extraction experiments. Each extraction experiment was performed for 45 min, and then the solvent was distilled under reduced pressure at $70^{\circ} \mathrm{C}$.

\subsection{Bleaching with Activated Bleaching Earth}

A certain amount of waste lubricating oil after extraction and sedimentation was accurately weighed into a three-necked flask. A relatively excess amount of activated bleaching earth was added to determine the effect of temperature on the degree of refining. After finding the optimum temperature, the amount of activated bleaching earth was adjusted to select the best proportion of activated clay. The stirring speed was fixed at $300 \mathrm{rpm}$, and the stirring time was fixed at $30 \mathrm{~min}$. After the stirring, the upper layer solution in the three-necked flask was placed in a beaker that was placed in a constant temperature drying oven at $70^{\circ} \mathrm{C}$ for $6-8$ hours. The final upper yellow liquid was the purified waste lubricating base oil.

The most intuitive color of waste lubricating oil is used as the evaluation standard. According to ASTM D1500-12, the final result is represented by the color code (i.e., chromaticity) of $0.5-8$. When the color of the oil exceeds 8 or more, it is necessary to add kerosene and then measure. This experiment simplifies the measurement method and treats it with 8 when the chromaticity exceeds 8 . The smaller the number, the lighter the color of the oil and the higher the degree of refining, and vice versa.

\section{Results and Discussion}

\subsection{Pretreatment with Thermal Settlement}

The contents of the mechanical impurities of the waste lubricating oil after thermal settling are shown in Figure 6. As the settling time increased, the five curves gradually become flat. After 24-h, it can be regarded as a horizontal line. Therefore, 24-h was considered as the optimal settling time. 


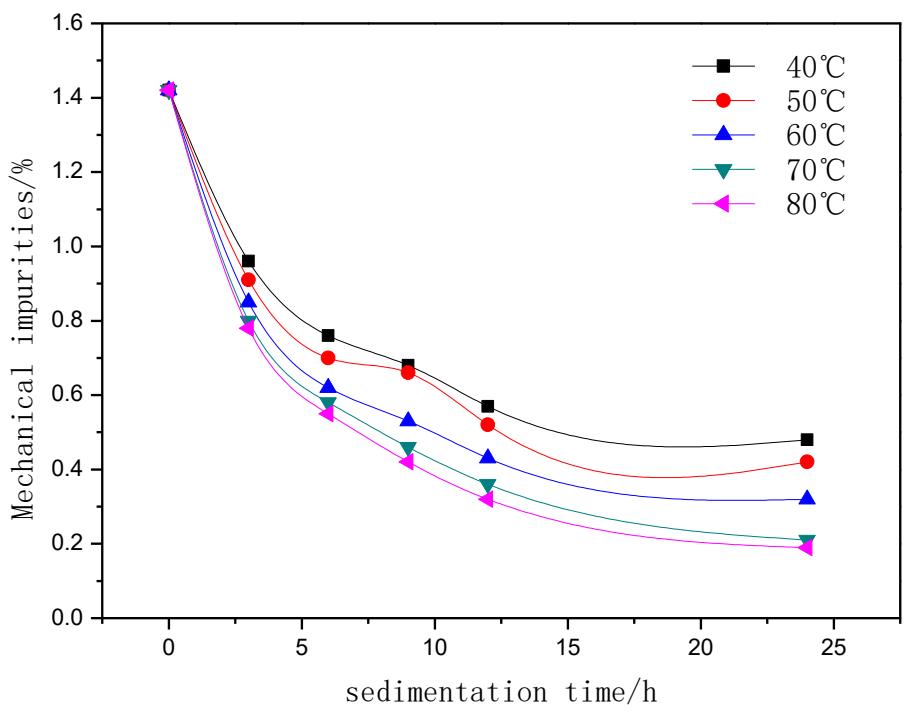

Figure 6. The effects of thermal settling temperature and time

The increase in the pretreatment temperature can significantly reduce the amount of mechanical impurities in the waste lubricating oil. At $70^{\circ} \mathrm{C}$ and $80^{\circ} \mathrm{C}$, the change of mechanical impurity content in waste lubricating oil was small. Because a higher temperature can increase the cost and the oxidative deterioration of oil, $70^{\circ} \mathrm{C}$ was considered as the optimal settling temperature. Subsequent tests used the waste lubricating oil after thermal settling at $70^{\circ} \mathrm{C}$ as experimental materials.

\subsection{Thermal Extraction and Sedimentation using Single Solvent}

Four solvents of isopropanol, n-butanol, isobutanol, and butanone were tested. The solvent was mixed with the waste lubricating oil in the ratio of $1: 1,2: 1,3: 1,4: 1$, and $6: 1$, and then heated at $50^{\circ} \mathrm{C}$ for $30 \mathrm{~min}$. The yield of waste lubricating oil was measured after purification. It was found that butanone had no obvious extraction effect and was basically miscible with lubricating oil. Figure 7 shows the relationship between the solvent to oil ratio and the yield of the recycled waste lubricating oil. When the ratio was higher than $3: 1$, there is no obvious yield change.

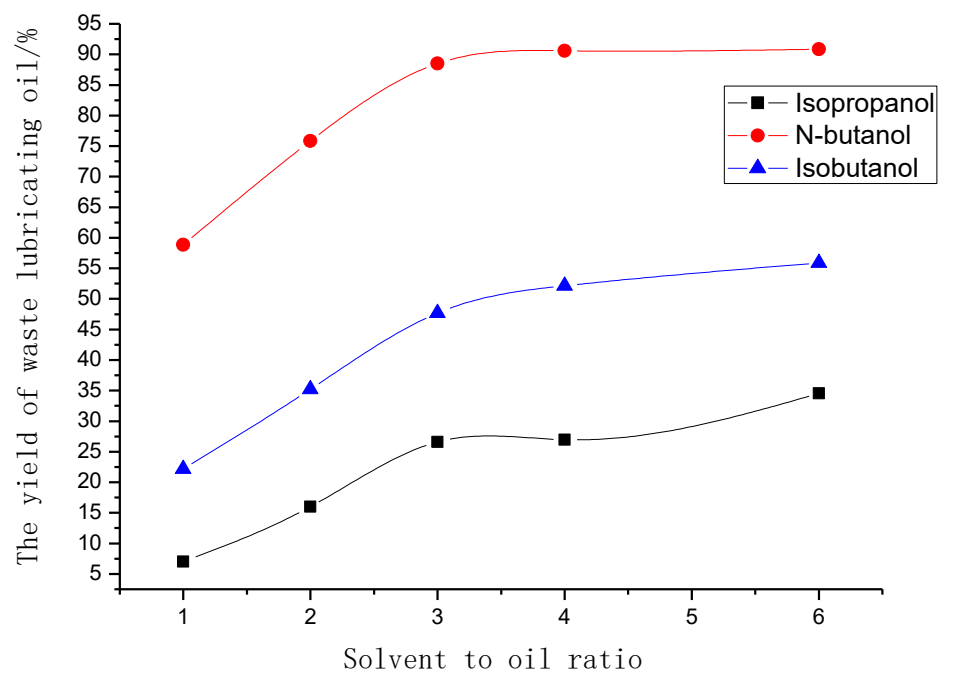

Figure 7. Effect of single solvent extraction on the yield of recovered lubricating oil 


\subsection{Thermal Extraction and Sedimentation using Double Solvents}

The selection of two solvents in the thermal extraction and sedimentation tests was based on the conclusions of single-solvent extraction. N-butanol has a strong ability to dissolve effective components of the waste lubricating oil, and isobutanol can reduce the ash content. Therefore, a combination of n-butanol and isobutanol was selected as the extractant.

Table 5. Analysis of variance of regression equation

\begin{tabular}{ccccccc}
\hline $\begin{array}{c}\text { Source } \\
\text { of } \\
\text { variance }\end{array}$ & $\begin{array}{c}\text { sum of } \\
\text { squares }\end{array}$ & $\begin{array}{c}\text { Degree } \\
\text { of } \\
\text { freedom }\end{array}$ & $\begin{array}{c}\text { Mean } \\
\text { squares }\end{array}$ & F value & P value $^{*}$ & Significance \\
\hline Model & 4928.83 & 9 & 555.84 & 32.77 & $<0.0001$ & significant \\
$\mathrm{A}$ & 3262.30 & 1 & 3262.30 & 195.22 & $<0.0001$ & significant \\
$\mathrm{B}$ & 425.01 & 1 & 425.01 & 25.43 & 0.0015 & significant \\
$\mathrm{C}$ & 31.13 & 1 & 31.13 & 1.86 & 0.2146 & \\
$\mathrm{AB}$ & 48.30 & 1 & 48.30 & 2.89 & 0.1329 & \\
$\mathrm{AC}$ & 59.99 & 1 & 59.99 & 3.59 & 0.1000 & \\
$\mathrm{BC}$ & 45.77 & 1 & 45.77 & 2.74 & 0.1419 & \\
$\mathrm{~A}^{2}$ & 323.95 & 1 & 323.95 & 19.39 & 0.0031 & significant \\
$\mathrm{B}^{2}$ & 188.81 & 1 & 188.81 & 11.30 & 0.0121 & significant \\
$\mathrm{C}^{2}$ & 436.26 & 1 & 436.26 & 26.11 & 0.0014 & significant \\
Residual & 116.97 & 7 & 16.71 & & & \\
Lack of & 86.04 & 3 & 28.68 & 3.71 & 0.1190 & Not \\
Fit & & & & & \\
Pure & 30.94 & 4 & 7.73 & & & \\
error & & & & & \\
Cor. total & 5045.81 & 16 & & & \\
& \multicolumn{7}{c}{$\mathrm{R}^{2}=0.9768$} & $\mathrm{R}_{\mathrm{ADJ}}{ }^{2}=0.9470$ & & \\
\hline
\end{tabular}

* The cut-off value for $\mathrm{P}$ value was 0.05 .

The results are shown in Figure 8. The multivariate quadratic regression fitting was performed, and the regression model of the process parameters was established. The coded regression equation for the specific yield $\mathrm{Y}$ is:

$\mathrm{Y}(\%)=82.95+20.19 \times \mathrm{A}+7.29 \times \mathrm{B}+1.97 \times \mathrm{C}-3.48 \times \mathrm{A} \times \mathrm{B}-3.87 \times \mathrm{A} \times \mathrm{C}-3.38 \times \mathrm{B} \times \mathrm{C}-8.77 \times \mathrm{A}^{2}-$ $6.70 \times \mathrm{B}^{2}-10.18 \times \mathrm{C}^{2}$

(Equation 1)

$\mathrm{A}, \mathrm{B}$, and $\mathrm{C}$, respectively, correspond to the coding values of the three factors, and the values are in the range of -1 to 1 . Therefore, the regression equation can be used instead of the test real point to calculate the experimental results.

The larger the $F$ value of the dependent variable, the greater the effect on the yield of the waste lubricating oil. It can be seen from Table 5 that the solvent to oil ratio (A) has the greatest influence on the yield of the waste lubricating oil, and the solvent ratio (B) has the second influence on the waste lubricating oil yield, and the temperature $\mathrm{C}$ has the least influence on the waste lubricating oil yield. 

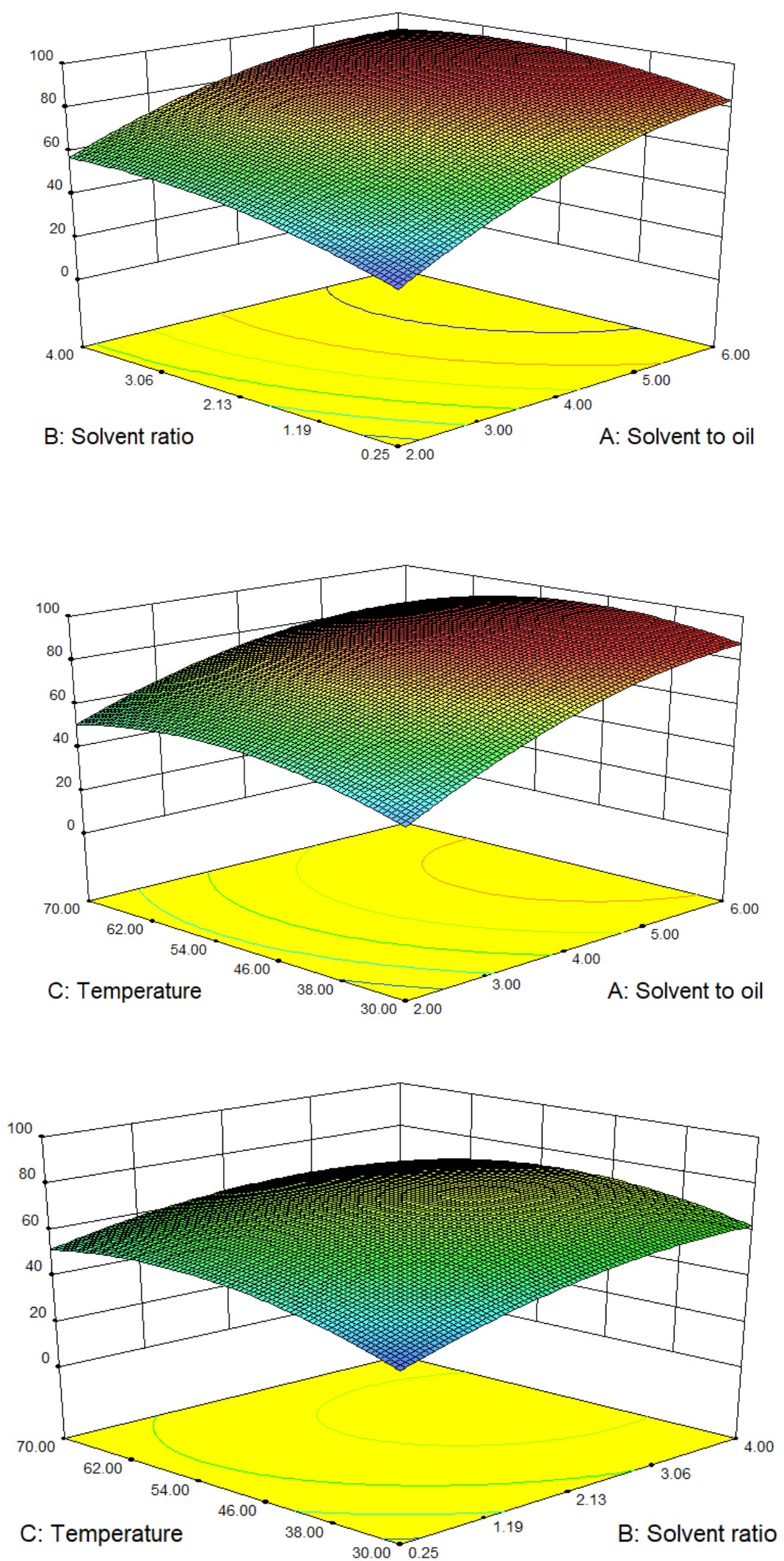

Figure 8. 3D response surface map of the thermal extraction and sedimentation using double solvents 
Table 6. Simulated optimal values for the two solvents

\begin{tabular}{ccccccc}
\hline Number & Solvent:Oil $\begin{array}{c}\text { Solvent } \\
\text { ratio }\end{array}$ & $\begin{array}{c}\text { Temperature } \\
\text { / } \mathrm{C}\end{array}$ & Yield/\% & Desirability \\
\hline 1 & 6 & 2.7 & 47.09 & 95.1234 & 0.364 & Selected \\
2 & 6 & 2.7 & 46.99 & 95.1227 & 0.364 & \\
3 & 6 & 2.7 & 47.94 & 95.1227 & 0.364 & \\
4 & 6 & 2.7 & 47.29 & 95.1222 & 0.364 & \\
5 & 6 & 2.7 & 46.85 & 94.1213 & 0.364 & \\
\hline
\end{tabular}

Based on Equation 1, the optimal conditions were calculated (Table 6). The optimal conditions were the ratio of solvent: waste lubricating oil of $6: 1, n$-butanol: isobutanol (vol ratio) of $2.7: 1$, temperature of $47.1^{\circ} \mathrm{C}$, and stirring time of $45 \mathrm{~min}$.

Table 7. Verification of the optimal conditions for the two solvents

\begin{tabular}{ccccc}
\hline Number & Solvent:Oil & $\begin{array}{c}\text { Solvent } \\
\text { ratio }\end{array}$ & $\begin{array}{l}\text { Temperature } \\
{ }^{\circ} \mathbf{C}\end{array}$ & Yield /\% \\
\hline 1 & 6 & 2.7 & 47.1 & 91.56 \\
2 & 6 & 2.7 & 47.1 & 89.65 \\
\hline
\end{tabular}

The optimal conditions were further experimentally verified. The yield of the waste lubricating oil product obtained under the above conditions was $90.60 \pm 1.35 \%$, and the viscosity at $40^{\circ} \mathrm{C}$ was $50.2 \mathrm{mPa} \cdot \mathrm{s}$, and the ash content was $0.23 \%$. Intuitively, the appearance color is lighter than the single extractant n-butanol as a solvent but still reddish brown. The acid value was $0.1 \mathrm{mg} \cdot \mathrm{KOH} / \mathrm{g}$.

Compared with the single extractant n-butanol, the yield of the recovered waste lubricating oil of the double-solvent extraction was slightly increased and the ash content decreased. In this test, the ratio of the solvent to the lubricating oil has the greatest influence, the solvent ratio has the secondary influence on the yield of the waste lubricating oil, and the temperature has the least effect. The optimal conditions experimentally verified were close to the simulated values, indicating that the method has certain persuasive power, and there are certain rules for each factor and response value.

\subsection{Bleaching with Activated Bleaching Earth}

The waste lubricating oil treated with thermal extraction and sedimentation was further purified by using activated bleaching earth. As shown in Figure 9, when the temperature was below $110^{\circ} \mathrm{C}$, the chromaticity of the waste lubricating oil did not change too much, and the activated bleaching earth obtained was dark yellow and did not play an adsorption role. When the bleaching temperature rose to $120^{\circ} \mathrm{C}$, the situation changed significantly, and the chromaticity of the waste lubricating oil dropped sharply. The bleaching earth obtained at this time was dark brown, and the colored materials in the oil were basically taken out. When the temperature was raised to $130^{\circ} \mathrm{C}$, the oil is 
already clear. Therefore, the choice of $125^{\circ} \mathrm{C}$ for the bleaching temperature, not only can get better oil, but also reduce heat and save costs.

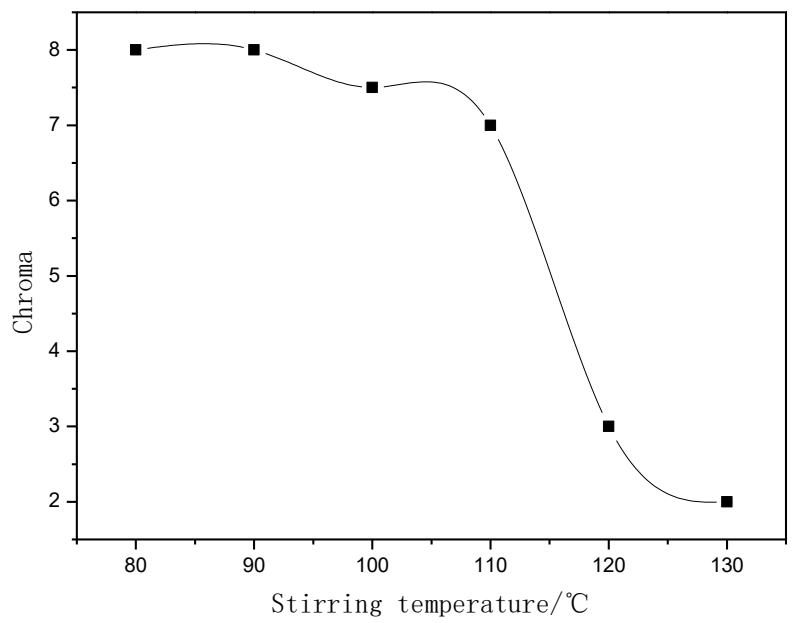

Figure 9. Relationship between temperature and oil color under excessive bleaching earth

After determining the bleaching temperature to be $125^{\circ} \mathrm{C}$, the relationship between the amount of bleaching earth added ( $\%$ of white soil quality: oil quality) and the color and the recovery rate of the final base oil were determined (Figure 10).

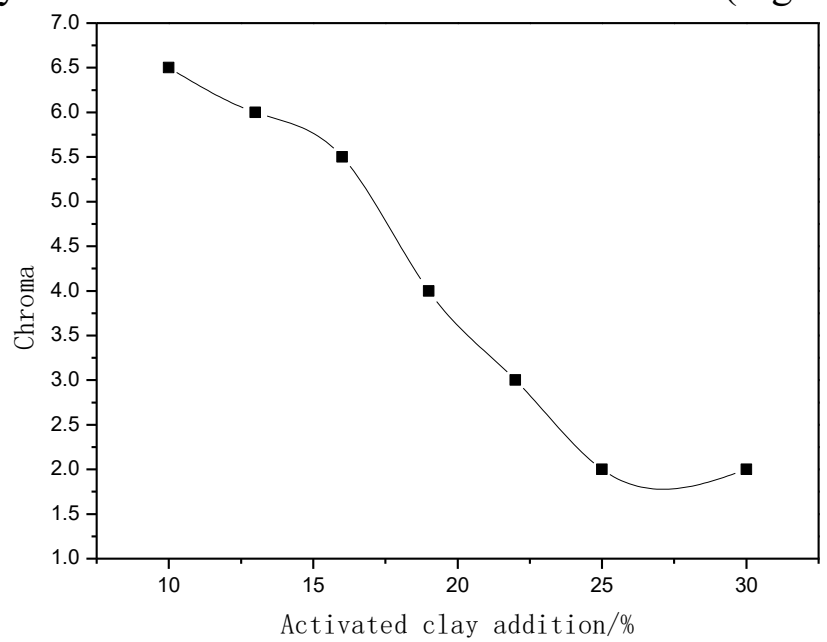

Figure 10. Effect of the amount of activated bleaching earth on the chromaticity

There is a strong correlation between the amount of activated bleaching earth and the degree of refining of the oil. As the amount of activated bleaching earth increased, the color of the waste lubricating oil gradually decreased. When the amount of activated bleaching earth reached $25 \%$, the color of lubricating oil was basically unchanged and became a relatively pure yellow transparent liquid. Figure 11 shows the change of appearance chromaticity of the waste lubricating oil with the addition of activated bleaching earth. 

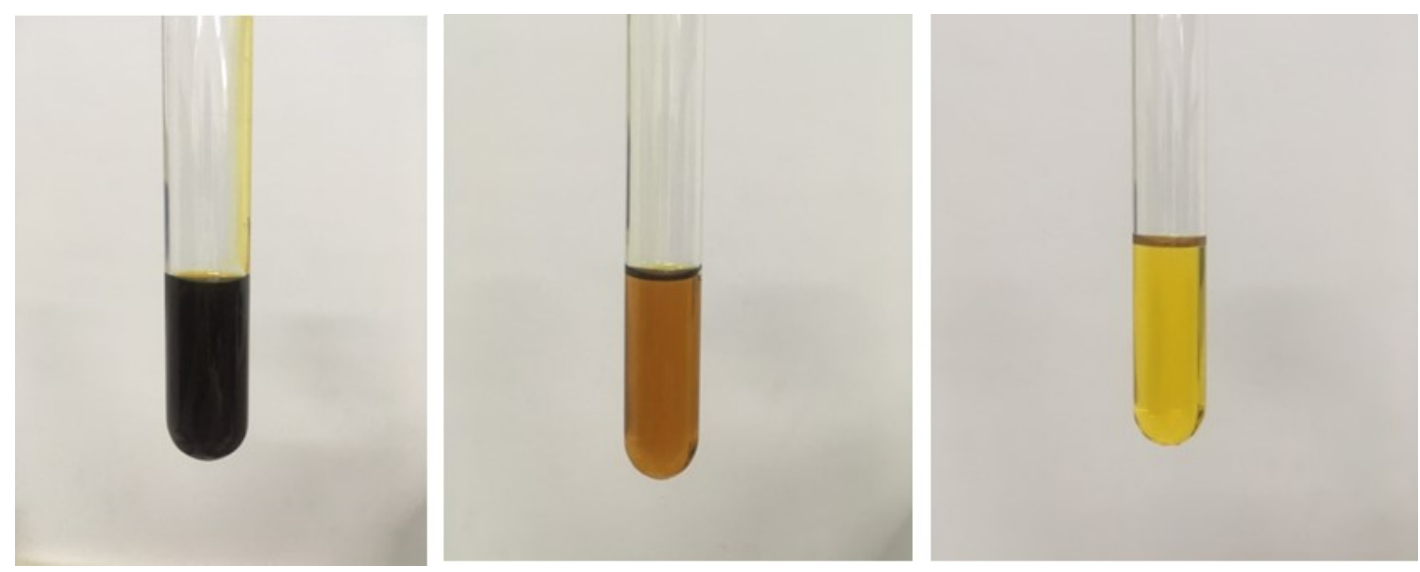

Figure 11. Appearance chromaticity change of waste lubricating oil

Left: the waste lubricating oil after thermal extraction and sedimentation; Middle: Treated with 15 $\mathrm{wt} \%$ of activated bleaching earth; Right: Treated with $25 \mathrm{wt} \%$ of activated bleaching earth

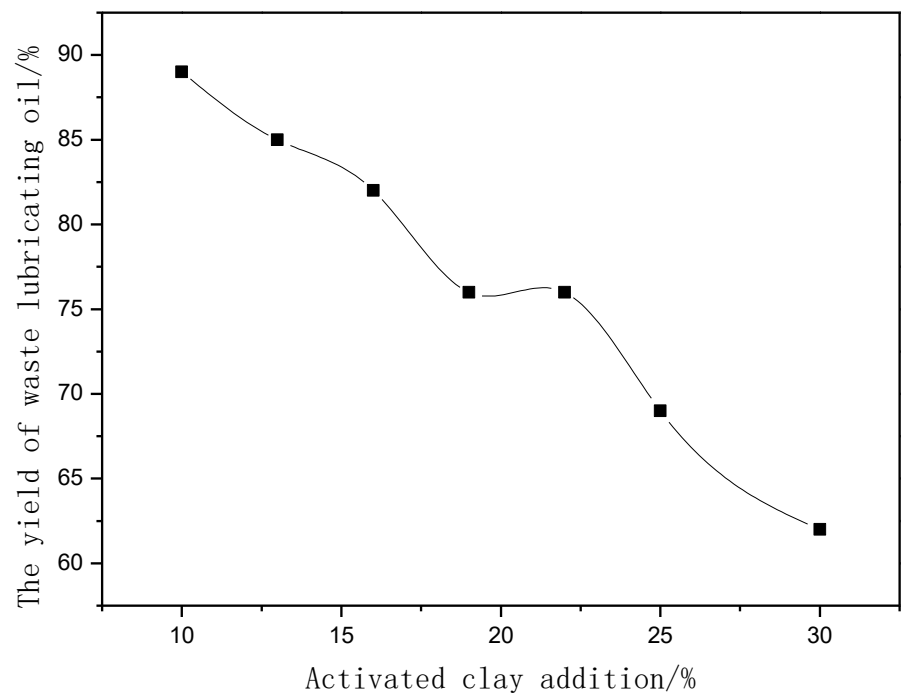

Figure 12. Effect of the amount of activated bleaching earth on the yield of waste lubricating oil

As can be seen from Figure 12, as the amount of activated bleaching earth increased, the yield of waste lubricating oil has been decreasing. On the one hand, too much bleaching earth will absorb a part of the oil, resulting in a decrease in the amount of recycled oil. On the other hand, due to the rise of the bleaching earth, the bleaching earth in the oil is difficult to settle in the process of the final thermal settling, and the separation speed is slow, resulting in oil turbidity and lowering the yield of the base oil.

In summary, in order to achieve a better purification effect, the amount of bleaching earth added was $25 \mathrm{wt} \%$, and the yield of refined lubricating oil was $69 \%$. 
Table 8. Properties of recycled lubricating oil

\begin{tabular}{cc}
\hline Properties & Value \\
\hline Dynamic viscosity@40 ${ }^{\circ} \mathrm{C}(\mathrm{mPa} \bullet \mathrm{s})$ & 24.0 \\
Kinematic viscosity@ $40^{\circ} \mathrm{C}\left(\mathrm{mm}^{2} / \mathrm{s}\right)$ & 21.8 \\
Viscosity index & 98.2 \\
Acid value $(\mathrm{mg} \cdot \mathrm{KOH} / \mathrm{g})$ & 0.04 \\
Moisture $(\%)$ & 0 \\
Mechanical impurities $(\%)$ & 0 \\
Ash content $(\%)$ & 0.02 \\
Pour point $\left({ }^{\circ} \mathrm{C}\right)$ & -14 \\
\hline
\end{tabular}

Table 9. HVI-100 Lubricant Standard

\begin{tabular}{|c|c|c|c|c|c|c|}
\hline $\begin{array}{l}\text { Kinematic } \\
\text { viscosity } @ 40^{\circ} \mathrm{C} \\
\left(\mathrm{mm}^{2} / \mathrm{s}\right)\end{array}$ & Appearance & Chromaticity & $\begin{array}{l}\text { Viscosity } \\
\text { index }\end{array}$ & $\begin{array}{l}\text { Pour } \\
\text { point } \\
\left({ }^{\circ} \mathrm{C}\right)\end{array}$ & $\begin{array}{l}\text { Acid value } \\
(\mathrm{mg} \cdot \mathrm{KOH} / \mathrm{g})\end{array}$ & $\begin{array}{c}\text { Ash content } \\
(\%)\end{array}$ \\
\hline $20 \sim 22$ & Transparent & 1 & 100 & $<-9$ & $<0.02$ & $<1.5$ \\
\hline
\end{tabular}

Comparing Table 8 with Tables 9 , it is found that the finally obtained lubricating oil sample basically conforms to the standard of the grade HVI-100 lubricating oil.

\section{CONCLUSIONS}

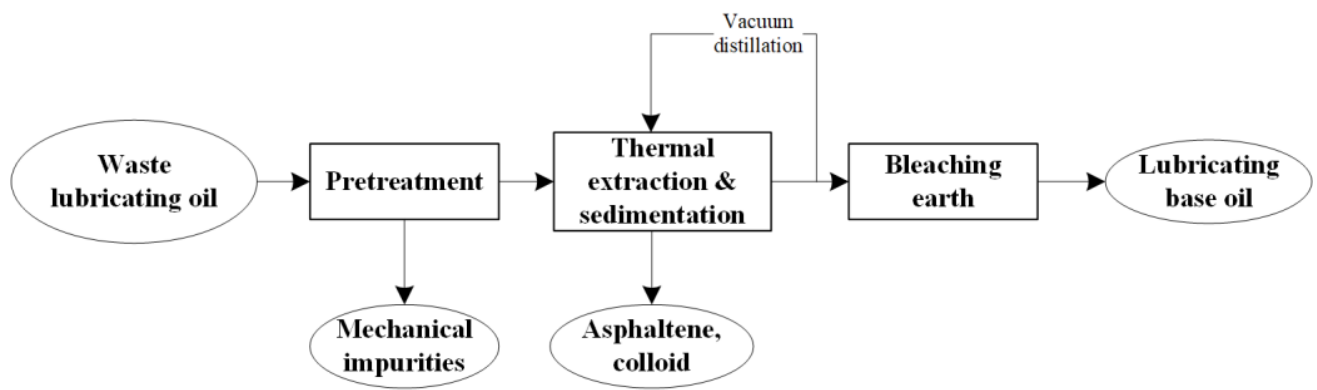

Figure 13. Green refining process of waste lubricating oil developed in this study

This paper proposes a new process for the regeneration of waste lubricating oil into lubricating base oil (Figure 13). The process has three stages. The first pretreatment stage removes mechanical impurities and large particulate matter from the waste lubricating oil via thermal settling. By comparing the effects of different temperatures and settling times on mechanical impurities, a reasonable settling time of $24 \mathrm{~h}$ and an optimum settling temperature of $70^{\circ} \mathrm{C}$ were found. The second stage is a thermal extraction and sedimentation process to remove impurities such as asphaltenes and gums. The thermal extraction and sedimentation process was carried out using a single solvent of isopropanol, n-butanol or isobutanol. It was found that the most economical ratio of the ratio of the solvent to oil was $3: 1$, the stirring time was $30 \mathrm{~min}$, and the operation temperature was around $50^{\circ} \mathrm{C}$. When using double solvents of n-butanol and isobutanol, the optimal conditions were the solvent to oil ratio of $6: 1$, the solvent ratio of $2.71: 1$, and the temperature of $47.1{ }^{\circ} \mathrm{C}$. Under these conditions, the yield of the oil experimentally 
obtained was $90.60 \pm 1.35 \%$. The third stage is bleaching with the activated bleaching earth, which adsorbs the pigments and the impurities such as colloid and asphaltene. The optimal temperature was determined as $125^{\circ} \mathrm{C}$ when the mass of bleaching earth was 25 $\mathrm{wt} \%$ of the oil mass. The refined lubricating oil is basically in accordance with the standard of the HVI-100 lubricating base oil. The total recovery rate of the process was about $63.5 \%$.

\section{ACKNOWLEDGMENTS}

This work is partially supported by Graduate Innovative Fund of Wuhan Institute of Technology (NO: CX2017129 and CX2018019) and the School of Chemical Engineering and Pharmacy at the Wuhan Institute of Technology.

\section{CONFLICTS OF INTEREST}

The authors declare that there is no conflict of interests regarding the publication of this paper.

\section{REFERENCES}

[1] An, J. (2017). Global lubricant market analysis and development forecast. Petroleum Products Application Research(3), 4-9.

[2] Wang, Q., Tao, C., and Yang, N. (2017). Automotive lubrication efficiency and long oil change period. Lubricating Oil(1), 1-6.

[3] Gao, X., and Tang, W. (2002). Replacing and Inspecting of Lubricant for Engine of Automobile. Shandong Metallurgy(4), 69-70.

[4] Speight, J. G. (2014). The chemistry and technology of petroleum, CRC press.

[5] Thom, R., Kollmann, K., Warnecke, W., and Frend, M. (1995). Extended oil drain intervals: Conservation of resources or reduction of engine life. SAE transactions, 706-718.

[6] Li, Y., Wu, J., Jiang, G., Wu, H., Huai, Y., and Zhao, W. (2016). Progresses in regeneration technologies for used lube oils. Petrochemical Technology(2), 244250.

[7] Liu, J., Zhang, Z., Gao, W., Lei, Y., and Bai, J. (2004). Technique for regenerating waste lubricating oil. CN1539936A.

[8] Liu, J. (2012). Solvent Extraction of Waste Gasoline Engine Oil-Flocculation Composite Regeneration Technology. China Academy of Machinery Science and Technology

[9] Whisman, M. L., Reynolds, J. W., Goetzinger, J. W., and Cotton, F. O. (1978). Process for preparing lubricating oil from used waste lubricating oil. Google Patents.

[10] Durrani, H. A., Panhwar, M. I., and Kazi, R. A. (2011). Re-Refining of waste lubricating oil by solvent extraction. Mehran University Research Journal of Eng. \& Tech, 30(2), 237-246. 
[11] Hsu, Y.-L., and Liu, C.-C. (2011). Evaluation and selection of regeneration of waste lubricating oil technology. Environmental monitoring and assessment, 176(1-4), 197-212.

[12] Lam, S. S., Liew, R. K., Jusoh, A., Chong, C. T., Ani, F. N., and Chase, H. A. (2016). Progress in waste oil to sustainable energy, with emphasis on pyrolysis techniques. Renewable and Sustainable Energy Reviews, 53, 741-753.

[13] Ackerman, A. H. (1929). Process for treating mineral oils. U.S. Patent No. 1,742,020.

[14] Audibert, F. (2006). Waste engine oils: rerefining and energy recovery, Elsevier, Amsterdam. DOI: 10.1016/B978-0-444-52202-3.X5018-8

[15] Zhang, B., Wu, J., Yang, C., Qiu, Q., Yan, Q., Li, R., Wang, B., Wu, J., and Ding, Y. (2018). Recent Developments in Commercial Processes for Refining BioFeedstocks to Renewable Diesel. BioEnergy Research, 11(3), 689-702. DOI: 10.1007/s12155-018-9927-y

[16] Zhang, B., and Seddon, D. (2018). Hydroprocessing Catalysts and Processes: The Challenges for Biofuels Production, World Scientific Publishing, Singapore. DOI: 10.1142/q0141

[17] Iwao, Y., Yamamoto, S., and Kunihiro, T. (1979). Method of preparing base stocks for lubricating oil. U.S. Patent 4,157,294.

[18] Manley, R. E., Mccarty, B. Y., and Gross, H. H. (1938). Solvent refining process. U.S. Patent 2,121,323.

[19] Sequeira Jr, A., Smith Jr, B. F., and Mead, T. C. (1981). Solvent refining process. U.S. Patent 4,294,689

[20] Gould, R. M., White, L. S., and Wildemuth, C. R. (2001). Membrane separation in solvent lube dewaxing. Environmental Progress, 20(1), 12-16. DOI: 10.1002/ep.670200110

[21] Lu, Y., Yang, J., and Wang, L. (2016). Study on Coloring Substances of Refined Lubricating Oil. Acta Petrolei Sinica, 32(1), 132-142.

[22] Alves dos Reis, M. (1991). Waste lubricating oil rerefining by extractionflocculation. 3. A pilot plant study. Industrial \& Engineering Chemistry Research, 30(11), 2449-2456.

[23] Mohammed, R. R., Ibrahim, I. A., Taha, A. H., and McKay, G. (2013). Waste lubricating oil treatment by extraction and adsorption. Chemical Engineering Journal, 220, 343-351.

Article copyright: (C) 2019 Jinlong Wu, Bo Li, Wei Wang, Shu Yang, Peng Liu, Bo Zhang, Changyan Yang, and Yigang Ding. This is an open access article distributed under the terms of the Creative Commons Attribution 4.0 International License, which permits unrestricted use and distribution provided the original author and source are credited. 